$16^{\text {th }}$ International Congress of Metrology, 02006 (2013)

DOI: $10.1051 /$ metrology/201302006

(C) Owned by the authors, published by EDP Sciences, 2013

\title{
New electromagnetic flowmeters for liquid metal
}

\author{
I. Velt ${ }^{\mathrm{a}}$, and Yu. Mikhailova \\ State Research Center "Niiteplopribor”, Moscow, Russia France
}

\begin{abstract}
The magnetic flowmeter in which inductor represents an electromagnet creating a pulse variable low-frequency magnetic field in the channel of a pipe is described. As a result of application of a variable magnetic field the influence of external hindrances of industrial frequency is completely eliminated; the influence of own induction, the capacitor hindrances essentially decreases, the influence of vortical currents is practically eliminated etc.
\end{abstract}

\section{Introduction}

Liquid metals (sodium, potassium, lithium, eutecticums of lead and bismuth, sodium and potassium) use as heatcarriers in nuclear power plants. Thus there is a requirement for flowmeters of the heat-carriers, which are capable to work in the conditions close to the extremal. A heat $\left(400-500^{\circ} \mathrm{C}\right)$, the difficult radiation conditions, and also extremely high requirements to reliability essentially limit possibilities in a choice of constructive decisions of devices.

At measurement of the liquid metals possessing electronic conductivity, the phenomenon of electrodes polarization is absent, hence it is possible to use a magnetic field created by a permanent magnet. The flowmeters for liquid metals flowmeters are accepted to name the magnetic flowmeters [1-2].

The conventionally designed electromagnetic flowmeter for liquid metals comprises a pipe made of stainless steel, two measuring electrodes welded onto the outer surface of the pipe wall, an inductor inducing a magnetic field within the working volume of the channel, and a measuring device. In the well-known designs, the inductor which is a constant magnet induces in the pipe channel a magnetic field which is directed perpendicularly to the plane passing through the line which connects the electrodes and the axis of the pipe [1], [2]. The liquid metals have electronic conductance, and the electrode polarization phenomenon does not exist, therefore a magnetic field induced by the constant magnet can be used in electromagnetic flowmeters.

At movement of liquid metal in a magnetic field, electric field is generated. The circulating currents are arises in liquid metal and in the pipe wall.As result the potential difference between electrodes are formed. This potential difference serves as a measure of the flow of liquid metal. The potential difference $e$ is calculated by the equation

$$
e=B D v=4 B Q_{0} / \pi D,
$$

where $B$ is magnetic induction, $D$ is a channel diameter, $v$ and $Q_{0}$ are respectively a mean velocity and a flow of the liquid metal.

The devices in question do not have trouble connected with noise and restrictions caused by electrode polarization. However, use of metals with high temperature and a high electric conductance leads to dependence of indications of a flowmeter from fluctuations of temperature of the measured medium. Due to the magnetic system being heated, the induction of the magnetic field decreased and, therefore, the potential difference $e$ decreased also. The corresponding reduction factor $k_{t}$ can be expressed by the formula

$$
k_{t}=1-\beta_{M}\left(t_{M}-t_{0}\right),
$$

where $\beta_{M}$ is a demagnetization factor the value of which is approximately $(2.6-2.8) 10^{-4} /{ }^{0} \mathrm{C}$ for magnets [1], $t_{M}$ is a mean temperature of the magnet, $t_{0}$ is an initial temperature of the magnet, which usually equals $20^{\circ} \mathrm{C}$. The temperature of the magnet in the operating mode of the flowmeter can reach $200{ }^{\circ} \mathrm{C}$ and over.

For certain types of magnetic system, experiments with an electromagnetic flowmeter show that with the temperature of the measured medium being $300{ }^{\circ} \mathrm{C}$ and the mean temperature of the magnet being $54{ }^{0} \mathrm{C}$ the factor $k_{t}=0,99$, and with increase in these temperatures respectively up to $400{ }^{\circ} \mathrm{C}$ and $75^{\circ}$ the factor $k_{t}$ decreases down to 0.985 . For controlling the influence of the temperature on the sensibility of the flowmeter with constant magnets, measures should be taken to provide the better cooling of the magnetic system, and the

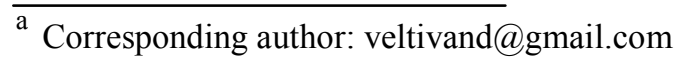


magnetic system itself should be made of hightemperature magnetic materials.

Besides, the flowmeter with a constant magnet is sensitive to the thermo-electromotive force arising in different components of the devices. If the material of the pipe and that of the electrodes are different, and if temperature difference exists between the electrodes as well, then parasitic thermo-electromotive force arises. To reduce it to an acceptable minimum, the electrodes and the pipe should be made of the same material. Moreover, the pipe units with the pairs of electrodes which induce at a temperature difference of $20{ }^{0} \mathrm{C}$ the thermoelectromotive force exceeding a certain minimum, e.g. in the order of $5 \mu \mathrm{V}$, should be rejected.

For the flowmeter with constant magnets, the arrangement of the electrodes is of significant importance. A vertical arrangement on a horizontal pipe is the most unfavourable. At a high temperature of metal, the strong air convection currents occur giving rise to the temperature difference of the electrodes and, therefore, to perceptible thermo-electromotive force.

Thus, for example thermo-electromotive force amounted to $0.2 \mu \mathrm{V}$ on a pipe of $25 \mathrm{~mm}$ diameter at a metal temperature of $300{ }^{0} \mathrm{C}$. [1]. Therefore, for flowmeters with constant magnets, it is advisable to arrange the electrodes in a horizontal plane, and preferably on a vertical pipeline.

Although electromagnetic flowmeters with constant magnets acquired general use due to their simple design, for the above reasons they do not satisfy with modern requirements for devices of the nuclear industry.

Their main disadvantages are as follows:

-low stability of the magnetic field in time,

-high temperature dependence of metrological

characteristics,

-low noise immunity,

-no possibility of controlling the mode of operation of the devices.

It is necessary to revise the design of the magnetic flowmeter, for the purpose of its modernisation.

\section{Electromagnetic flowmeter}

As is known, the inductor is the main constructive knot of a flowmeter. The inductor defines the basic operational characteristics of a flowmeter: sensitivity, stability, metrology, power consumption, adaptability, weight, etc. In State Research Center "Niiteplopribor", Moscow, has been developed a magnetic flowmeter in which индуктор represents an electromagnet creating a pulse variable lowfrequency magnetic field in the channel of a pipe.

The experience of applying an alternating magnetic field in electromagnetic flowmeters of general industrial type for measuring flow of liquids with ionic conductivity has been used in designing the devices. High stability of flow measurement and negligible temperature dependence of metrological characteristics have been attained by applying an alternating magnetic field and a special algorithm for signal. Like in many flowmeters of general industrial type, a low-frequency of the pulsed bipolar magnetic field of the inductor which is an electromagnet has been applied.

The processing of a signal from the electrodes is performed as follows: during the transient process of switching polarity of the magnetic field the signal does not change, reading of the signal from the electrodes is taken only at the points of time when the magnetic field becomes settled. Besides, the summation of signals measured in the presence of a positive and a negative pulse of the magnetic field makes it possible to eliminate thermo-electromotive force and other parasitic signals the origin of which is not bound up with the magnetic field of the inductor. As the magnetic field polarity changes, Foucault currents occur in different structural components of the flowmeter. The period of transient process is not informative and it is excluded from the measurement process.

As a result of application of a variable magnetic field the influence of external hindrances of industrial frequency is completely eliminated; influence of own induction, capacitor hindrances and of the thermoelectromotive force decreases essentially , influence of vortical currents, drift of a signal and influence of noise is practically eliminated; etc. The application of the variable magnetic field decreases has allowed to solve a question about diagnosing of a flowmeter during functioning and about the control of the metrological characteristics of the flowmeter.

On fig. 1 the scheme of a design of an offered electromagnetic flowmeter for the liquid metal is presented.The electromagnetic flowmeter of liquid metal consists of a pipe 1 executed from stainless steel without an electroinsulating cover, and of the inductor, consisting of the magnet 2 of the $\mathrm{C}$-shaped forms with pole pieces 3 and of the induction coil 4. For fastening of the inductor to a pipe there are apertures, and axes of apertures coincide with axes of pole pieces 3 . the inductor is fixed to a pipe by means of the racks 6 inserted into above mentioned apertures of poles. Racks are welded on a pipe 1 in places of crossing of a forming pipe with an axis of poles 5 . From a rack opposite side are screwed up by nuts 7. Two electrodes 8 are welded to an external surface of a pipe on a line which is perpendicular a line connecting the centres of poles. The casing divides a flowmeter design on two zones, on the high-temperature zone in which there is a pipe with liquid metal, and the lowtemperature zone in which there is an induction coil and the central part of the magnet. The site of a pipe located near to a casing is wound round with a tape from asbestos or a basalt fabric.

Thanks to such design the raised reliability of the device and protection of the induction coil against thermal radiation of a pipe with liquid metal is provided.

A new design is proposed for fastening the inductor to the pipe. In all well-known flowmeters with constant magnets the inductor is connected to the pipe at the pipe periphery, which increases the overall dimensions, shunts a part of the signal and partially distorts the induced electric field in the pipe channel. In the new devices the fastening elements connecting the inductor to the pipe are located at those points on the surface of the central part of the pipe, in which the electric potential of the induced 
magnetic field is always equal to zero, regardless of velocity of the liquid metal flow streaming along the channel. In this case the fastening element does not lead to shunting a flowmeter signal, does not disturb the distribution of currents in the pipe walls and does not change their value.

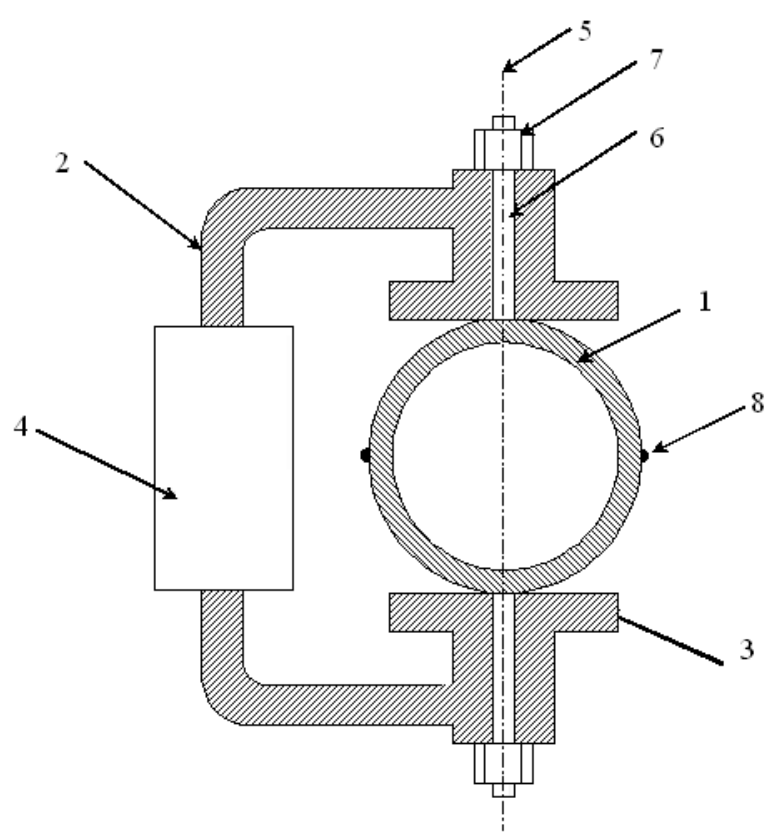

Fig. 1

Using an alternating magnetic field inductor made it possible to solve the problem of in-service operation diagnostics of the flowmeter and control of its metrological characteristics.

Metrological and technical characteristics of a flowmeter the following:

- the measured medium - liquid sodium;

- the temperature of the measured medium from $350^{\circ} \mathrm{C}$ to $525^{\circ} \mathrm{C}$;

- pressure of the measured medium no more than 1,0 $\mathrm{MPa}$;

- temperature of air surrounding the primary transducer from $20^{\circ} \mathrm{C}$ to $90{ }^{\circ} \mathrm{C}$ at relative humidity from 45 to $80 \%$;

- air temperature in a place of placing of the electronic transducer from 5 to $40^{\circ} \mathrm{C}$ at relative humidity to $80 \%$.

Flowmeters are developed for modifications, for diameters of conditional pass (DN) and for ranges of measurement of an flow are specified in table 1 .

Table 1

\begin{tabular}{|c|c|c|c|}
\hline \multirow{2}{*}{ Modification } & \multirow{2}{*}{$\mathrm{DN}, \mathrm{mm}$} & \multicolumn{2}{|c|}{ Volume flow, $\mathrm{m}^{3} / \mathrm{h}$} \\
\cline { 3 - 4 } & & $\mathrm{Q}_{\min }$ & $\mathrm{Q}_{\max }$ \\
\hline IRMU-1-25 & 25 & 0,03 & 3 \\
\hline IRMU -1-40 & 40 & 0,12 & 12 \\
\hline IRMU -1-80 & 80 & 1,3 & 130 \\
\hline IRMU-1-100 & 100 & 1,5 & 150 \\
\hline
\end{tabular}

Limits of an admissible basic error of a flowmeter is no more than $\pm 2 \%$ from the upper range of measurements.
The mass of the component parts of the flowmeter is no more than:

- $11 \mathrm{~kg}$ for the primary transducer,

- $8 \mathrm{~kg}$ for the electronic transducer.

The mean lifetime of the flowmeter is no less than 15 years.

The mean-time-between-failures is no less than $100,00 \mathrm{~h}$, the faultness probability of the flowmeter over $8,000 \mathrm{~h}$ which corresponds to the above is no less than:

- $\quad 0.99$ for the primary transducer;

- 0.96 for the electronic transducer.

The flowmeter has:

- DC analogue output signal of $4 \mathrm{~mA}$ to $20 \mathrm{~mA}$;

- digital indication of the measured flow on the display board within the measurement range of $(0 \ldots 100) \%$;

- RS485 code interface.

The flowmeter with a diameter $100 \mathrm{MM}$ has been tested on down-pour plant for liquid sodium. Results of tests are resulted in table 2.

Table 2

\begin{tabular}{|c|c|c|c|l|}
\hline \multicolumn{2}{|c|}{ Down-pour plant } & \multicolumn{3}{|c|}{ Flowmeter } \\
\hline $\begin{array}{c}Q_{p}, \\
\mathrm{~m}^{3} / \text { hour }\end{array}$ & $\begin{array}{c}\text { Accidental } \\
\text { error, } \%\end{array}$ & $\begin{array}{c}Q_{f}, \\
\mathrm{~m}^{3} / \text { hour }\end{array}$ & $\begin{array}{c}\text { Accidental } \\
\text { error, } \%\end{array}$ & $\begin{array}{l}\text { Basic } \\
\text { error, } \%\end{array}$ \\
\hline 75,3220 & 0,03 & 75,6567 & 0,02 & $\begin{array}{c}\gamma=0,22 \\
\delta=0,44\end{array}$ \\
\hline 98,4534 & 0,02 & 99,0181 & 0,01 & $\begin{array}{c}\gamma=0,38 \\
\delta=0,57\end{array}$ \\
\hline 98,4364 & 0,02 & 98,9141 & 0,01 & $\begin{array}{c}\gamma=0,31 \\
\delta=0,49\end{array}$ \\
\hline 1,1711 & 0,18 & 1,2083 & 0,10 & $\begin{array}{c}\gamma=0,02 \\
\delta=3,18\end{array}$ \\
\hline 2,24552 & 0,13 & 2,28856 & 0,09 & $\begin{array}{c}\gamma=0,03 \\
\delta=1,91\end{array}$ \\
\hline 3,17498 & 0,05 & 3,23111 & 0,05 & $\begin{array}{c}\gamma=0,04 \\
\delta=1,77\end{array}$ \\
\hline
\end{tabular}

Here $Q_{p}$-the indications of down-pour plant, $Q_{f}$ - the flowmeter indications, $Q_{h}$ - the maximum value of the flow, $Q_{h}=150 \quad \mathrm{~m}^{3} /$ hour $\quad, \gamma=\frac{Q_{f}-Q_{p}}{Q_{h}} \times 100 \%$, $\delta=\frac{Q_{f}-Q_{p}}{Q_{p}} \times 100 \%$.

It is as a result received, that dependence of flowmeter indications $Q_{f}$ from indications of down-pour plant $Q_{p}$ can be described as follows:

$$
Q_{f}=1.0054 Q_{p}-Q_{0}, Q_{0}=0.0159 \mathrm{~m}^{3} / \text { hour }
$$

\section{References}

1. P. P. Kremlevsky. Multiphase Flow Rate Measurement. (EDP Mashinostroyenie, Leningrad, 1982)

2. N. I. Loginov. Electromagnetic Liquid Metal Flow (EDP Energoizdat, Moscow,1981) 